\title{
Has Sub-centre Policy Produced Sub- centres? An Evaluation of Melbourne's Urban Spatial Planning since 1996
}

\section{Jennifer Day, Weiqing Han, Amy Boxi Wu and Jiarui Zheng ${ }^{1}$}

\section{Abstract}

This study evaluates Melbourne's longstanding 'activity centres' (AC) policies—the first study to do so. It strongly suggests that, across the Melbourne metropolitan area, AC policies have had no effect on the propensity of people to work near their homes. The findings are robust to a number of validity hazards. The study does not warrant a wholesale abandonment of $A C$ planning, but does signal that we may wish to question how we are currently going about transforming 'places' into 'centres'. For $A C$ policies to be successful, designation as a 'centre' may be necessary, but is not sufficient.

\section{Introduction}

This study tests whether metropolitan activity centre (AC) planning actually produces activity centre-like behaviours among commuters. State planning authorities in Victoria, Australia, have been attempting to facilitate the development of urban sub-centres located outside Melbourne's central business district (CBD) since at least 1981 (Melbourne and Metropolitan Board of Works, 1981, pp. 57-9, 63-4). Since then, five successive metropolitan plans have been developed and implemented, with the latest released in 2016. One significant feature of all of these plans has been significant attention paid to developing ACs-concentrated

1 University of Melbourne, jday@unimelb.edu.au. Sophie Sturup and Jennifer Day conceived the first iterations of this study in 2012. This study could never have happened without the sustained work and support of the Australian Urban Research Infrastructure Network (AURIN) and its staff, including Serryn Eagleson, Chris Pettit, Jack Barton and Bob Stimson. AURIN also funded the data assembly. Thanks also to Pietro Calogero and Stefan $\mathrm{Al}$, along with Dr Sturup, for many useful comments on the writing. 
nodes of activity that include jobs, entertainment and residential uses. The logic of this spatial planning strategy is that non-CBD ACs will house people and jobs to promote polycentric urban forms, reducing driving distances and trips to the urban core. In the 2014 strategy document, Plan Melbourne, this was described as the '20-minute neighbourhood' concept (Victorian Department of Transport, 2014, p. 11).

A problem with these spatial policies is that they have never been evaluated for their long-term efficacy. This implies that Victorian state governments since at least 1981 have been making spatial policy in metropolitan Melbourne with little empirical evidence that those policies are creating the urban fabric that is the stated intention of the plans.

We do not contest that spatial planning is important for metropolitan regions. Our worry is that the particular type of planning that occurs in Melbourne may not be producing the outcomes that planners envision. Rather than a coherent vision that has persisted over time, Melbourne's metropolitan planning shifts frequently. The statutory agency responsible for the implementation of metropolitan plans (which are developed by state government planning ministries) has changed names and responsibilities twice since 2008, when it was originally the Growth Areas Authority, charged with developing a strategic approach for new suburbs outside the urban core. In 2013, the agency's name was changed to the Metropolitan Planning Authority, and it was tasked with implementation of metropolitan planning for the whole metropolitan region including the urban core. In 2016, the agency's name and scope were again changed: now called the Victorian Planning Authority, it is responsible for state-wide planning implementation, including in the Melbourne metropolitan area.

Melbourne's AC policies provide a good natural experimental framework from which to test the viability of the policies. The metropolitan spatial plans have seen frequent changes in language and location. For instance, the 1995 Living Suburbs strategy (Victorian Department of Planning and Development, 1995) designates 12 'Major Mixed Use Activity Centres', while the 2002 Melbourne 2030 (Victorian Department of Infrastructure, 2002a, 2002b) designates 25 'Principal Activity Centres' and the 2014 Plan Melbourne designates 9 'Metropolitan Activity Centres'. The plans vary in the levels of ACs designated, from the CBD to 'National Employment Clusters' to 'Major Car-Based Shopping Centres' to 'Specialised Activity Centres'-all which vary with each plan. This shift in spatial designation provides the opportunity to observe places that have changed designations, acquired designation later and lost designation in later plans. 


\section{Background}

In this literature review, we start with the different attitudes towards $\mathrm{AC}$ policies in Melbourne from government and academic perspectives, the disconnect between which highlights a need for long-term evaluative study of metropolitan policies in Melbourne. We then turn to international discourses that reveal a disconnect between policy outcomes and academia, illuminating a need for robust methodologies in studying AC impacts. Lastly, we justify jobs-housing balance (JHB), as used in this study, as an effective measurement to examine the impact of AC policies.

\section{The need for analysis of AC policy}

Urban planning researchers and practitioners have long been concerned with the relationship between metropolitan spatial planning and economic outcomes associated with where people work, live and travel. Much of the discussion revolves around whether the decentralising of urban activities into ACs, or 'sub-centres', can influence the clustering of employment, housing or commercial activities (see Day et al., 2015). In Victoria, the designation of ACs has been implemented as a component policy of metropolitan planning documents since 1954, although the objectives of these policies have fluctuated over time.

Despite the popularity of ACs in government policymaking, the impact of these policies are not well understood in the literature. Analysing Census-derived journeyto-work (JTW) data, Gordon et al. (1991) find that from 1980 to 1985, there was an association between sub-centre development and decreased commuting time, as residents moved closer to their places of work. However, these findings are contradicted by Cervero and Landis (1991), who find that the suburbanisation of jobs resulted in a threefold increase in commuting time, as measured in vehicle miles travelled. As our previous AC research has posited (Day et al., 2015), statistical associations between urban structures and commuting activities are limited by the inherent heterogeneity of specific urban centres. This is further exacerbated by lack of clear consensus about metrics to study AC outcomes and the subsequent difficulty of aligning findings to applied policy. This study attempts to address this barrier, by proposing a quantitative methodology based on an existing metric: the jobs-housing ratio (JHR). This ratio is adopted and modified in our study, which will be discussed in detail in the following sections.

A further source of disconnect between academic discourse and policy comes from the difficulty in comparing the process of sub-centre development across geographies. While urban planning has traditionally been dominated by North American and European discourses, Melbourne's distinctly top-down and 'modernist' (Mees, 2003, p. 297) approaches to metropolitan planning may in fact draw lessons from the increasing body of literature concerning AC models in Chinese cities. 
For example, Monaghan et al. (2015) draw links between heavily regulated AC models in Shanghai and applications for ACs in Australia - the research posits that AC policy can successfully direct commercial activity into concentrated centres without negative impacts on retail competition and heterogeneity. This is in contrast to relatively neoliberal discourses in Australia to 'remove planning and zoning regulation and encourage out-of-centre development' (Productivity Commission, 2011, in Monaghan et al., 2015, p. 11).

Only two papers study temporal dynamics in Melbourne: our previous study (Day et al., 2015) which analysed two waves of data in 2006 and 2011 to evaluate the impact of AC policies in employment clustering, and a report (Goodman et al., 2010) looking at housing construction data from 1990 to 2007. There are obvious temporal gaps in these two studies: Day et al. (2015) fail to access data before the introduction of Melbourne 2030, and Goodman et al.'s report (2010) was limited to the 5 years following the plan's release. Day et al. (2015) is the only Melbourne study to attempt to identify whether sub-centring has occurred outside of planned ACs, although these sorts of studies have been conducted in other metropolitan regions like Los Angeles: for example, Giuliano and Small (1991).

\section{A proxy for evaluating AC outcomes: Jobs-housing balance (JHB)}

Our literature review thus far suggests that AC policies in Victoria lack substantial directives about how to achieve their proposed objectives, and that there is a lack of adequate data for which outcomes can be measured. Recognising these deficits, we propose the JHB ratio as an appropriate metric for quantifying $\mathrm{AC}$ benefits and providing a way for policymakers to benchmark outcomes to objectives.

The JHB metric posits that balanced and sustainable urban sub-centres (or ACs) should have a comparable number of jobs to available housing units within geographic proximity (Schleith et al., 2016). This metric is commonly calculated as a ratio of jobs and housing (JHR), employing Census-derived travel data (origindestination data, JTW, etc.). A key benefit being that such data is relatively accessible at the AC's relevant municipal geography (Giuliano, 1991; Peng, 1997). The JHR, an operationalisation of JHB, has been widely adopted in a number of studies (such as Giuliano, 1991; Cervero, 1995; Peng, 1997; Lin et al., 2015). Cervero (1995), for instance, suggests that JHB is indicative of other characteristics of ACs, namely an area's self-containment; i.e. the extent to which the built form 'allows many to live, work, shop and recreate within a community or defined geographical area' (Cervero, 1995, p. 1136).

This line of thinking aligns with that of the Victorian Government, which acknowledges the significance of JHB in its policymaking. Plan Melbourne contains strategies designed to promote employment, residential proximity and 
shorter commute times. Consequently, this study is formed on the premise that $\mathrm{JHB}$ is an appropriate proxy for AC outcomes. We acknowledge that AC policies could have other impacts-as is stated in Melbourne 2030 and Plan Melbourne, key objectives of ACs include stimulating business activities, boosting community activities, improving accessibility to ACs by public transport, etc. However, both plans have attached considerable importance to spatial distribution of activities. In Melbourne 2030, the primary objective of ACs is to reduce car dependency by concentrating activities in highly accessible places. Similarly, Plan Melbourne stressed the redistribution of jobs outside the central city under its Direction 1.2 (Victorian Department of Infrastructure, 2002a; Victorian Department of Transport, 2014). In the 'Indicators' section, we describe how we improve the JHR to provide a better indicator of JHB.

\section{Hypotheses}

If metropolitan AC policies are effective, then those ACs should be inducing people to live and commute locally-more so than people who do not live in the influence area of an AC. Compared with places that are not influenced by an AC, then, a place in the influence area of an AC should have:

Hypothesis 1: A higher proportion of trips originating in the area, ending in the same area or nearby.

Hypothesis 2: Stronger growth in the proportion of trips originating in the area, ending in the same area or nearby.

We are left, then, to operationalise the ideas of 'nearby' and 'influence area', as well as JHB. We take up these ideas in the Indicators section.

\section{Data and quasi-experimental design}

A significant contribution of this study is that it assembles datasets that have never been assembled and analysed in a peer-reviewed analysis. These are:

1. Panel data on JHB in the Melbourne metropolitan area, assembled from the Census of Population and Housing;

2. Historical panel data on AC locations, derived from examination of individual spatial plans.

To enable a historical look at our problem, we use data from the Australian Census of Population and Housing, which reports place of work data on origin and destination of commute. The Australia Census has occurred every five years since 
1981, and metropolitan plans were released in 1981, 1995, 2002, 2014 and 2016. However, the 2016 Census does not contain geographies that concord with prior Censuses, so it is not possible yet to study the impacts of the 2014 and 2016 spatial plans with the Census. We have been unable to match 1981, 1986 and 1991 origindestination-formatted commute data with commensurate spatial geographies due to missing unique identifiers. This leaves us with the quasi-experimental design shown in Figure 1 to test the hypotheses we outline above:

\begin{tabular}{ccccccc}
$\mathrm{X}_{1981}$ & $\mathrm{X}_{1995}$ & $\mathrm{O}_{1996}$ & $\mathrm{O}_{2001}$ & $\mathrm{X}_{2002}$ & $\mathrm{O}_{2006}$ & $\mathrm{O}_{2011}$ \\
\hline $\mathrm{X}_{1981}$ & & $\mathrm{O}_{1996}$ & $\mathrm{O}_{2001}$ & & $\mathrm{O}_{2006}$ & $\mathrm{O}_{2011}$
\end{tabular}

Figure 1. Timeline of quasi-experimental design

Source: Authors.

where $\boldsymbol{O}_{Y Y Y Y}$ is an observation of JHB in a given year, from the Census data, and $X_{Y Y Y Y}$ represents the policy intervention in a given year.

Using the concept of 'influence area' described below, we operationalise the control group as being those places with no or less area influenced by ACs. We use the Statistical Local Area (SLA) as our unit of analysis. We do this for two major reasons. First, the next-smallest area at which origin-destination data are reported in the Australian Census is the Destination Zone (DZN). DZNs are problematic from a longitudinal perspective because of the lack of concordance we mention above. DZNs are also much smaller than SLAs: in 1996, there were 76 SLAs in the area that is currently designated as the Melbourne metropolitan area according to Plan Melbourne. There were 3,157 DZNs. This brings us to the second reason why we use SLAs. Because of its larger geography, the SLA represents a meaningful geography within which a person might commute. We concord all data back to a common set of SLAs produced in 1996 so that data across common areas could be compared. Some of these SLAs were added to the metropolitan area after 1996, but urban designation in 1996 does not matter for our analysis.

\section{Indicators}

This section defines the indicators developed for the analysis, including rationales for their selection.

\section{JHB measures}

The simplest framings of JHB simply use a ratio of trip origins to trip destinations, with trip origins a proxy for the number of workers living in an area, and trip destinations a proxy for the number of jobs in an area. However, such framings do not account for conditions where there is both a large inflow and a large outflow 
of commuters to and from a place. It is possible that most workers who live in an SLA leave the SLA for work, and that other workers arrive from other places. Another problem with this framing is that any commute out of the SLA creates a jobs-housing imbalance-even if the worker commutes a few metres over an SLA boundary. This is a common problem with area-based studies that we mitigate by operationalising the idea of 'nearby'.

Regarding nearby-ness, because we have origin and destination data, we can do better than a simple metric. We can allow places that are close to a given SLA to contribute to that SLA's JHB, and for that effect to attenuate with distance. Equation 1 shows the equation we could use to estimate the JHB of a given SLA.

$J H B O_{i}=\sum_{j=1}^{n} \frac{O D_{j i}}{O_{i * D I S T_{i j}}}$

where $O D_{j i}$ is the number of trips originating in $j$ and ending in $i$, for $j \neq i, D I S T_{i j}$ is the linear distance (in kilometres) between the centroids of SLA and SLA; and for $j=i, D_{I S T_{i j}}=1$. This is an SLA's JHBO.

Essentially, Equation 1 measures the propensity of people who live in a given SLA or nearby to also work in that SLA. However, there is a converse measurement of AC behaviour that is also important: that people who work in a given SLA also live in that SLA. We therefore compute another JHB measurement and use it in the analysis, which reflects this converse relationship. This relationship is shown in Equation 2:

$J H B D_{j}=\sum_{i=1}^{n} \frac{O D_{i j}}{D_{j} * D I S T_{i j}}$

where $O D_{i j}$ is the number of trips originating in $i$ and ending in $j$; for $i \neq j, D I S T_{i j}$ is

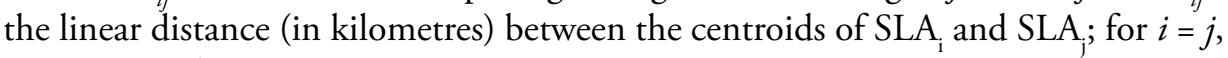
$D I S T_{i j}=1$. This is an SLA's JHBD.

The measurements contained in Equations 1 and 2 signal a higher JHB if the measure is larger.

\section{ACs and influence areas}

The AC locations are derived from the examination of the relevant metropolitan plans. We use the same dataset developed and used by Day et al. (2015) to locate the ACs in the metropolitan space. Our hypotheses describe the 'influence area' of an AC. We reproduce their logic in Table 1, which shows the alignment of class types. 
Table 1. Classifications of $A C$ types across plans

\begin{tabular}{|c|c|c|c|c|}
\hline & Class 1 & Class 2 & Class 3 & Class 4 \\
\hline Plan Melbourne (2014) & $\begin{array}{l}\text { Central city } \\
\text { (CBD) }\end{array}$ & $\begin{array}{l}\text { Metropolitan } \\
\text { activity centre }\end{array}$ & Activity centre & $\begin{array}{c}\text { National } \\
\text { employment } \\
\text { cluster }\end{array}$ \\
\hline Melbourne 2030 (2002) & $\begin{array}{l}\text { Central activities } \\
\text { district (CBD) }\end{array}$ & $\begin{array}{c}\text { Principal activity } \\
\text { centres }\end{array}$ & $\begin{array}{l}\text { Major activity } \\
\text { centres }\end{array}$ & $\begin{array}{c}\text { Specialised } \\
\text { activity centres }\end{array}$ \\
\hline \multirow[t]{2}{*}{ Living Suburbs (1995) } & \multirow[t]{2}{*}{$\begin{array}{l}\text { Central city } \\
\text { (CBD) }\end{array}$} & $\begin{array}{l}\text { Major mixed use } \\
\text { activity centre }\end{array}$ & \multirow[t]{2}{*}{$\begin{array}{l}\text { Major growth } \\
\text { area centre }\end{array}$} & $\begin{array}{c}\text { Major education } \\
\text { and employment } \\
\text { centre }\end{array}$ \\
\hline & & $\begin{array}{l}\text { Major car-based } \\
\text { shopping centre }\end{array}$ & & $\begin{array}{l}\text { Manufacturing } \\
\text { and production } \\
\text { concentrations }\end{array}$ \\
\hline $\begin{array}{l}\text { Metropolitan Strategy } \\
\text { Implementation (1981) }\end{array}$ & $\begin{array}{c}\text { Central } \\
\text { Melbourne }\end{array}$ & District centre & $\begin{array}{c}\text { Potential district } \\
\text { centre }\end{array}$ & $\begin{array}{c}\text { Industrial } \\
\text { concentrations }\end{array}$ \\
\hline $\begin{array}{l}\text { Scenario } 1 \text { influence } \\
\text { area (km radius) }\end{array}$ & 2.0 & 0.8 & 0.4 & 1.0 \\
\hline $\begin{array}{l}\text { Scenario } 2 \text { influence } \\
\text { area ( } \mathrm{km} \text { radius) }\end{array}$ & 3.2 & 2.4 & 1.6 & 2.4 \\
\hline
\end{tabular}

Source: Authors' summary of Day et al. (2015).

The next step was to operationalise each AC's influence area, which are also shown in Table 1. This is the area around an AC within which we can expect to see AC-like behaviour such as increased JHB. We use the above-mentioned AC centroids and draw buffers at these linear distances to identify the influence area of an AC. We describe how we deal with complexities like overlapping influence areas in the section below, 'AC influence areas'. For robustness, we conduct a sensitivity analysis using two scenarios for impact area:

- Scenario 1: small impact areas

- Scenario 2: large impact areas.

For Scenario 1, we follow Day et al. (2015), identifying influence areas according the AC classes. They cite a government publication that provides guidance on planning ACs, Structure Planning for Activity Centres, Practice Notes (Victorian Department of Planning and Community Development, 2010), which notes that neighbourhood ACs should be supported by improved walkability within 400 to 800 metres from the AC's core. With 400 metres as a starting point, Day et al. (2015) identify influence areas for each AC class as per the Scenario 1 influence area in Table 1. 
For Scenario 2, we enlarge the impact areas established by Day et al. (2015). Our review of the planning documents concurs with Day et al. (2015), indicating that they provide very little guidance for constructing impact areas. We use Melbourne 2030's language about neighbourhood ACs as a starting point (Victorian Department of Infrastructure, 2002b):

Design the centre and residential areas so that at least 60 per cent of dwellings and activities are within 400 metres safe walking distance from a bus stop, or 800 metres from a railway station (p. 45).

'Neighbourhood activity centre' is an AC type with smaller scale than Class 3 ACs. In Melbourne 2030, there are 900 neighbourhood ACs whose impact we do not include in this study due to their numerousness, their very small scale and their presence only in the 2002 plan-not in the 1995 plan or the 2014 plan.

Outside of neighbourhood walkability, the plans provide no guidance on impact areas around ACs. We start with this 800 -metre buffer for neighbourhood ACs as a benchmark, and apply proportionally larger buffers to AC designations that would presumably be more impactful. Thus, in Table 1, Class 3 ACs are given an impact radius of twice the 800 -metre radius. Class 2 are ACs are given an impact radius of 1.5 times the neighbourhood ACs, and Class 1 (the $\mathrm{CBD}$ ) has quadruple the impact radius compared with neighbourhood ACs. National employment clusters and their equivalents also receive a buffer of 2.4 kilometres in radius. We do note the arbitrariness of these impact areas. However, as we describe in the Findings section, the size of the impact areas appears to matter very little in the actual JHB computations. Regardless of whether we use large or small impact areas (Scenarios 1 or 2), there is very little link between the metropolitan policies and JHB.

\section{AC influence indicators}

This section describes the SLA-based indicators of 'influence'. Because the unit of analysis (SLA) is a spatial polygon, there are a number of ways in which we can operationalise the impact of ACs on that polygon. For instance, we could conceive indicators that reflect whether the centroid of an $\mathrm{AC}$ is located in a given polygon. This ignores the possibility that an AC could have its centroid in one polygon but significantly affect JHB in a neighbouring polygon. We could measure whether an $\mathrm{AC}$ touches a polygon, assigning a dummy value of one if the $\mathrm{AC}$ is touched by the buffer around an AC and a value of zero if it is not. However, this process would count SLAs that are 90 per cent covered by an AC the same as SLAs that are 1 per cent covered - though they are very likely not influenced similarly. Also, because of the large number of ACs outlined in the policy, there is often overlap between the influence area of one $\mathrm{AC}$ and another. 
Our final indicator is the proportion of total land area of an SLA covered by AC influence areas, with overlapping areas double-counted as necessary. This means that the indicators for influence can range from zero to more than 100 per cent. We do not apply weights to the different AC classes, since their influence is reflected in their spatial scope (400-2,000 metres) and applying a weight would amount to double-counting.

\section{Analysis methods}

We produce a variety of summary statistics and statistical tests, and we also estimate a series of simple panel models to test the hypothesis that there is a relationship between AC designation and JHB. We use a suite of models in order to rule out the possibility that an inappropriate (biased or inefficient) estimator is the reason we see no link between AC designation and AC-like behaviour among commuters. We start with a simple ordinary least squares (OLS) model with errors clustered on the SLA - a process that partially accounts for the persistence of JHB in an SLA over time. We next estimate the fixed effects estimator, which allows us to control for timeinvariant effects such as distance from the urban core. We estimate a second model, an OLS model with a lagged Coverage variable, again with clustered error terms. We also estimate two fixed effects models with lagged coverage variables: a standard fixed effects model with bias correction, which Kiviet (1995) argues is a suitable way to handle dynamic panel bias (correlation between the dependent variable and lagged dependent variables used as regressors) and a generalised method of moments model. None of these models contains time-variant control variables such as public transportation accessibility or average age of housing stock, which could have some explanatory power. We leave out further controls because the purpose of this paper is to explore these simple relationships between $\mathrm{AC}$ designation and JHB — not to uncover the forces driving the creation of JHB. For readability, we do not fully describe those models here, nor do we report the detailed findings. The models are more fully specified and justified in a working paper that is currently under review (Day et al., 2017).

\section{Findings}

This section describes the data we have compiled, presents a descriptive narrative of the story it tells and reports the results of the regression analysis. We conclude that AC policy has had no observed effect on JHB. 


\section{AC profiles}

The total number of AC designations has increased over the four policies, from 20 in 1981 to 45 in 1995. In 2002, there was a boom in the number of ACs, with 115 centres designated that are of comparable size to those in the other plans. In 2002, Melbourne 2030 additionally designated 900 'neighbourhood ACs', which we do not include in our analysis because their scale is too small. There were 133 ACs designated in 2014.

\section{Coverage}

This section describes the Coverage data. Figures 2 to 4 illustrate the influence area in the map for the 1981, 1995 and 2002 policies. As more ACs were designated in the 1995 and 2002 plans, the total influence area has increased over time. Thirteen SLAs were excluded as they were at the edge of the study area and not officially designated as part of the metropolitan area in 1981. Another five SLAs were excluded for missing data of JHB indicators in some years. This leaves us with 69 SLAs of the 76 SLAs in metropolitan Melbourne in 1996.

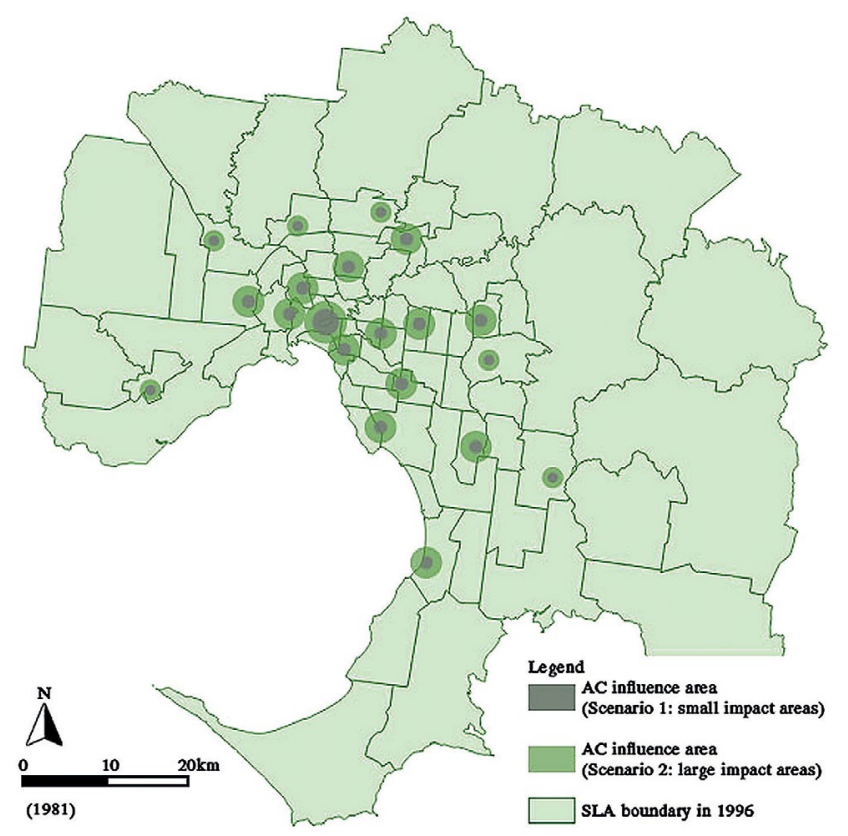

Figure 2. AC influence area for the 1981 policy

Source: Authors' summary of Melbourne and Metropolitan Board of Works (1981, pp. 57-9, 63-4). 


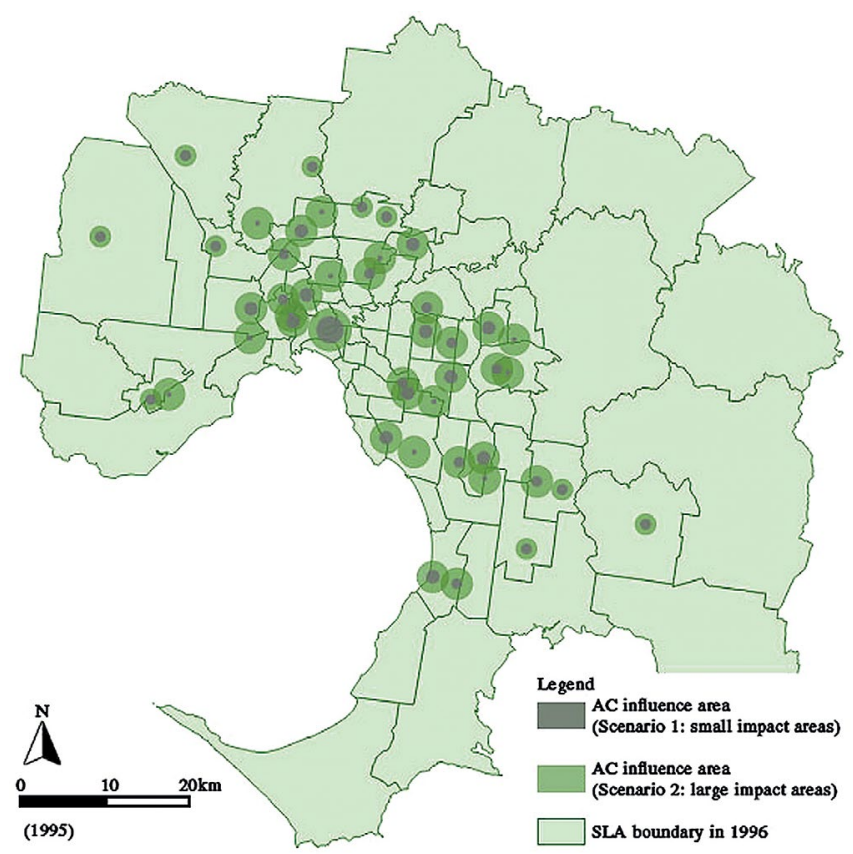

Figure 3. AC influence area for the 1995 policy

Source: Authors' summary of Victorian Department of Planning and Development (1995, p. 68).

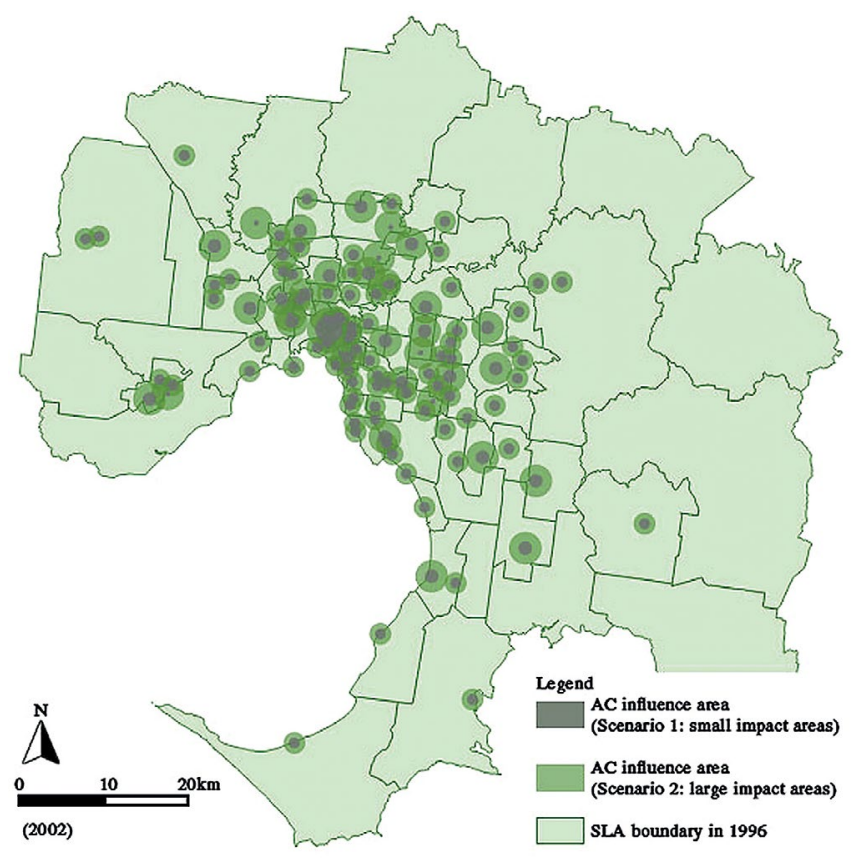

Figure 4. AC influence area for the 2002 policy

Source: Author's summary of Victorian Department of Infrastructure (2002a, pp. 46-51). 
The total land area of metropolitan Melbourne was 7,453,381,888 $\mathrm{m}^{2}$ in 1996. For constancy of comparison, we use the 1996 SLA boundaries throughout this analysis, collapsing all data to these geographic units. In 1981, under the impact scale of Scenario 2, 4.09 per cent of the total land was within an AC influence area. That proportion rose to 7.78 per cent in 1995 and then to 11.40 per cent in 2002. Impacts were smaller for Scenario 1, ranging from 0.87 per cent in 1995 to 3.2 per cent in 2002.

In aggregate, the Coverage ratio of SLAs increased dramatically between 1981 and 2002. Because of the growth in the total AC numbers, there was overlap in the $\mathrm{AC}$ areas. We dealt with this by double-counting the overlapping areas of two or more ACs.

\section{JHB profiles}

This section describes the change in JHB in the Census data years. Table 2 shows that the mean of JHBO (see Equation 1) declined marginally in 2006 and 2011 from 1996 and 2001 levels. The range and standard deviation, growing in 2011 and decreasing afterwards, suggests an overall decline in JHB in the metropolitan area. For JHBD (see Equation 2), the overall profile is fluctuating but relatively steady.

Table 2. Summary of statistics of JHBO/JHBD, 1996-2011

\begin{tabular}{|l|l|l|l|l|l|l|}
\hline Indicator & Year & $\mathrm{N}$ & Mean & Std. Dev. & Median & Range \\
\hline \multirow{4}{*}{ JHBO } & 1996 & 69 & 0.320 & 0.0993 & 0.302 & $0.113-0.642$ \\
\cline { 2 - 7 } & 2001 & 69 & 0.321 & 0.107 & 0.301 & $0.0482-0.710$ \\
\cline { 2 - 7 } & 2006 & 69 & 0.313 & 0.0933 & 0.299 & $0.145-0.660$ \\
\cline { 2 - 7 } & 2011 & 69 & 0.309 & 0.0946 & 0.292 & $0.159-0.691$ \\
\hline \multirow{3}{*}{ JHBD } & 1996 & 69 & 0.416 & 0.168 & 0.363 & $0.114-0.798$ \\
\cline { 2 - 7 } & 2001 & 69 & 0.424 & 0.162 & 0.404 & $0.118-0.788$ \\
\cline { 2 - 7 } & 2006 & 69 & 0.415 & 0.157 & 0.377 & $0.144-0.775$ \\
\cline { 2 - 7 } & 2011 & 69 & 0.427 & 0.163 & 0.402 & $0.152-0.845$ \\
\hline
\end{tabular}

Source: Authors' computations on Australian Census data.

Figure 5 shows a selection of profiles using the JHBD metric and provides a few examples of SLAs that take on this pattern. (We do not provide JHBO as the trends are similar.) We note that we have not systematically identified profile types, as this study is about large overall trends and their relationship to AC influence. Our purpose here is to illustrate the features of the data we use for the analysis. The statistical tests we report later in this section seek to understand whether JHB variations systematically follow changes in AC designation. Among the profile types we identify are (with sample SLAs named in parentheses): 
- Early increase-stronger increase in the earlier years of the study period followed by weaker change in later years (Hume).

- Early decrease-stronger decrease in the earlier years of the study period followed by weaker change in later years (Melton).

- Late change - a change in the trend near the end of the study period (BrimbankSunshine).

- Steady_varies over a narrow band of JHBD values (Cardinia South).

- Steady rise—steadily increasing JHBD values (Melbourne non-CBD, nonSouthbank/Docklands).

- Flatline-JHBD values are virtually unchanged over the study period (Kingston North, Nillumbik South).

- Temperamental-JHBD values vary seemingly without pattern and over a wide band (Whittlesea North, Nillumbik).

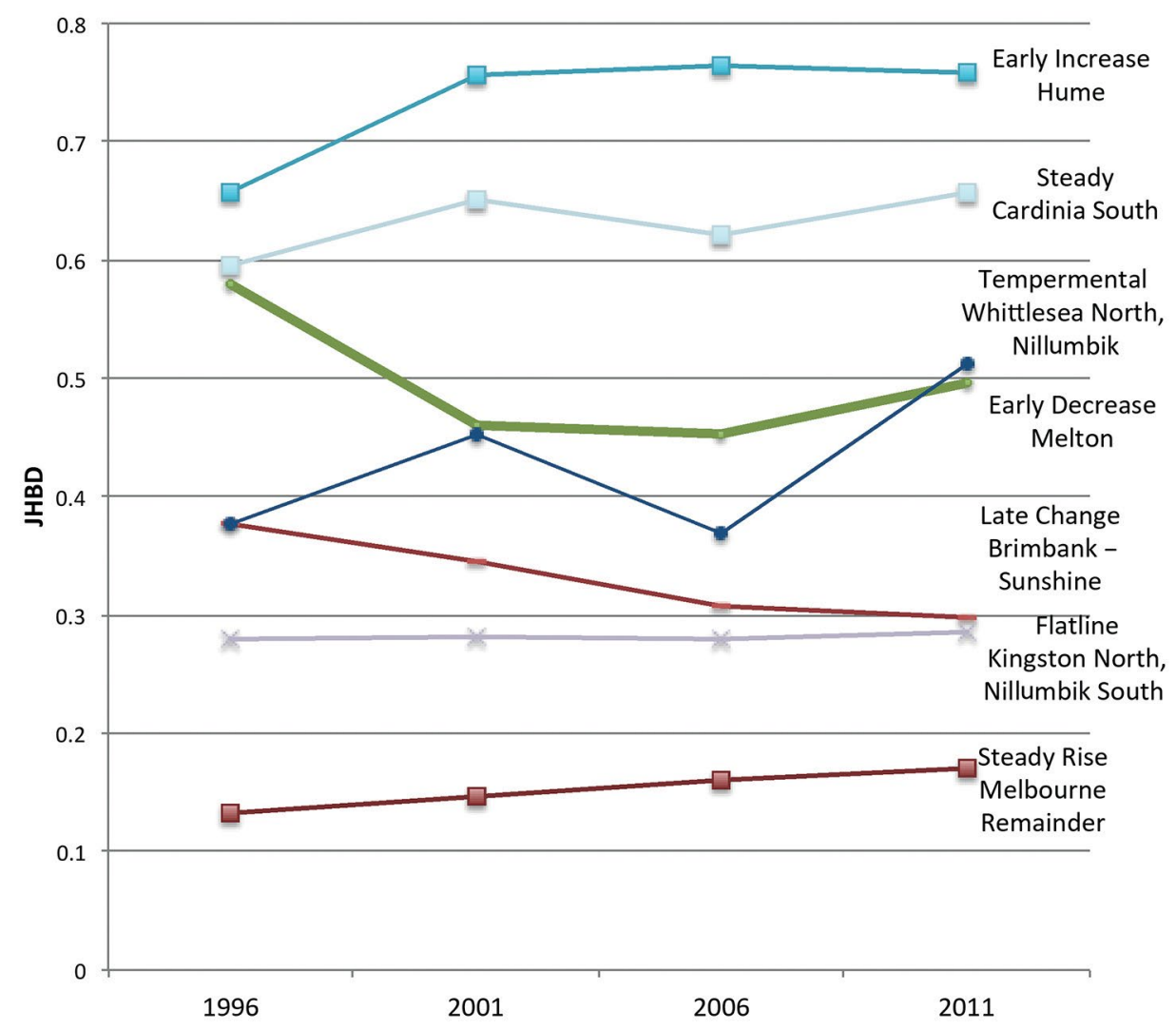

Figure 5. JHBD profiles, 1996-2011

Source: Author's computations on Australian Census data. 


\section{Association between $\mathrm{AC}$ influence and JHB}

This section tests for the effect of any Coverage at all on JHB measures. We divide the 69 SLAs into two groups according to whether they were touched by AC influence area-that is, whether any portion of the SLA is within an AC influence area. Before 2002, there were 52 SLAs influenced by an AC. After 2002 and Melbourne 2030, 10 more SLAs were touched by an AC influence area. This section suggests that $\mathrm{AC}$ policies have produced at best no effect on JHB and at worst an effect that is counterproductive. Either way, the observed statistical direction of the relationship does not suggest policy efficacy.

We use a two-sample t-test to test whether there is significant difference between the mean JHBO and JHBD of AC influence and non-AC influence groups in each policy year. Table 3 shows the mean JHBD and JHBO values for SLAs in each category. In every test year, the mean $\mathrm{JHBO}$ of the $\mathrm{AC}$ influence group is marginally lower than non-AC influence group-suggesting that SLAs with no AC influence have higher jobs-housing balance. This relationship is only statistically significant in the year 2001, and the p-value is slightly higher than 0.05 . These results do not suggest a strong and sustained difference between influenced and non-influenced SLAs in any of the data years.

Table 3 also shows the same relationships for JHBD, and the results are even more troubling. In each data year, the JHB of the non-influenced group is higher than the JHB of the influenced group. This effect is exactly the opposite of the effect we would expect if the AC policies were increasing JHB. These differences in the two metrics also appear in the regression results in the next section, suggesting that precision in the measurement of JHB is important for evaluating AC policies. There, we also address the possibility that ACs may have been created in order to improve $\mathrm{JHB}$ in low-JHB places.

Table 3. Two-sample t-test of JHBO/JHBD

\begin{tabular}{|c|c|c|c|c|c|c|c|c|}
\hline \multirow[b]{2}{*}{ Year } & \multicolumn{4}{|c|}{ JHBO } & \multicolumn{4}{|c|}{ JHBD } \\
\hline & 1996 & 2001 & 2006 & 2011 & 1996 & 2001 & 2006 & 2011 \\
\hline $\begin{array}{l}\mathrm{N} \text { of SLAs } \\
\text { (influenced/ } \\
\text { non-influenced) }\end{array}$ & $52 / 17$ & $52 / 17$ & $62 / 7$ & $62 / 7$ & $52 / 17$ & $52 / 17$ & $62 / 7$ & $62 / 7$ \\
\hline AC influence & $\begin{array}{l}0.311 \\
(0.0125)\end{array}$ & $\begin{array}{l}0.307 \\
(0.0125)\end{array}$ & $\begin{array}{l}0.308 \\
(0.0119) \\
\end{array}$ & $\begin{array}{l}0.306 \\
(0.0121) \\
\end{array}$ & $\begin{array}{l}0.370 \\
(0.0190)\end{array}$ & $\begin{array}{l}0.392 \\
(0.0186)\end{array}$ & $\begin{array}{l}0.391 \\
(0.0179) \\
\end{array}$ & $\begin{array}{l}0.402 \\
(0.0181) \\
\end{array}$ \\
\hline Non-AC influence & $\begin{array}{l}0.347 \\
(0.0299)\end{array}$ & $\begin{array}{l}0.365 \\
(0.0342)\end{array}$ & $\begin{array}{l}0.358 \\
(0.0315)\end{array}$ & $\begin{array}{l}0.341 \\
(0.0345)\end{array}$ & $\begin{array}{l}0.558 \\
(0.0433)\end{array}$ & $\begin{array}{l}0.521 \\
(0.0487)\end{array}$ & $\begin{array}{l}0.621 \\
(0.0556)\end{array}$ & $\begin{array}{l}0.652 \\
(0.0617) \\
\end{array}$ \\
\hline Difference & $\begin{array}{l}0.0360 \\
(0.0276) \\
\end{array}$ & $\begin{array}{l}0.0575 \\
(0.0292) \\
\end{array}$ & $\begin{array}{l}0.0504 \\
(0.0370) \\
\end{array}$ & $\begin{array}{l}0.0350 \\
(0.0378) \\
\end{array}$ & $\begin{array}{l}0.188^{\star * *} \\
(0.0413)\end{array}$ & $\begin{array}{l}0.129^{\star \star \star} \\
(0.0426)\end{array}$ & $\begin{array}{l}0.229^{\star \star \star} \\
(0.0564) \\
\end{array}$ & $\begin{array}{l}0.250^{\star \star \star} \\
(0.0577) \\
\end{array}$ \\
\hline p-value & 0.1969 & 0.0531 & 0.1772 & 0.3576 & 0.0000 & 0.0034 & 0.0001 & 0.0001 \\
\hline
\end{tabular}

(Std. Err.); ${ }^{\star \star \star} \mathrm{p}<0.01$

Source: Authors' computations on Australian Census data. 


\section{Regression analyses}

This section describes the regression results, wherein we test the associations between Coverage and JHB. We first ran the regressions on the full set of SLAs. To test the possibility that low-JHB places may have been designated as places to receive AC designation in order to encourage JHB there, we ran a second set of regressions to look at only those places that had the most to gain from AC policies.

We do not present the statistical findings in this section. To keep the paper interesting to a wide audience, we have placed the regressions in a working paper (Day et al., 2017).

The full-sample regression results reveal no supportable relationship between Coverage and JHB. The results further suggest that $\mathrm{AC}$ policies have not produced either form of JHB (JHBO or JHBD) in those areas where JHB was not present initially. This finding is robust across model specifications and estimators used, so the effect is likely to be a true effect and not a result of statistical misspecification or poorly constructed indicators. We test a variety of model specifications, including models using the Scenario 1 and Scenario 2 impact areas and models for both Scenarios with fixed and lagged effects. The estimated models for Scenario 1 (small impact areas) and Scenario 2 (large impact areas) are qualitatively identical. That is, they are identical across all models estimated in sign and are very similar in the magnitude of the significances and model fit statistics. This suggests that the results are robust to changes - even significant changes—in the Coverage indicator.

The results for the initially underperforming (low-JHB) places are even more discouraging for AC policies. For these SLAs, the results suggest that AC policies have at best produced no effect, and at worst have somehow been counterproductive to JHB.

\section{Conclusions: Can AC policies be reinvigorated?}

Our findings unfortunately suggest that ACs policies in Melbourne are not promoting JHB. The JHBO tests are very clear: AC policies appear to have no effect on the propensity of people to work nearby their homes. The JHBD tests are harder to interpret, but they also suggest that AC policies have at best had no effect on encouraging people to live nearby their workplaces. At worst, AC-influenced places are worse off when it comes to JHB. Moreover, those places that did not start with AC-like behaviour have not grown to be more AC-like. The State of Victoria dedicates a government agency to develop metropolitan activity spatial plans, including AC plans, and AC planning has been going on since 1981. This is a significant resource base dedicated to planning that may not be achieving the desired outcomes. 
Our results are robust to a number of threats to validity. Given the quasiexperimental design and the sensitivity analysis, the results appear to be robust to both unspecified temporal effects and variations in the Coverage measurement. Our results also do not suffer from bias associated with the spatial definitions or spatial autocorrelation, as we have incorporated nearby-ness into the indicators for JHB. Our results observe the system back to 1996 , so it is not likely that AC effects have yet to materialise to a sufficient degree to be observable. In short, these findings are ones that policymakers should take seriously, especially given the resources that are devoted to metropolitan planning in Victoria.

What this research demonstrates is there is probably more to creating vibrant ACs than just designating places as ACs. We also know that the state government provides varying levels of consultation and support in structure planning for localities that seek to cooperate with the metropolitan plans and develop ACs. Our study does not reflect significant issues like local capacity and uptake of offered assistance-again due to a lack of available data on these processes. We propose extensive follow-up research to confirm our findings and expand the analysis to include queries on what it is that drives AC success. Some next steps may be to conduct comparisons of places that have been given support, like Dandenong, to places that have received less support. Unfortunately, changes to the 2016 Census formats mean that further follow-up will not be straightforward using the same indicators. The upcoming 2016 Census data does not have the SLA as a proffered spatial unit of analysis. Australia's public agencies should consider a continuity of data that allows for ongoing, refreshed examination of the policy outcomes, but at present appears to have no plans to do so.

Our study does not imply that a wholesale abandonment of AC planning is warranted. It does, however, signal that we may wish to question how we are currently going about making places into centres. We hope that future studies will demonstrate that some AC planning has indeed been fruitful under some conditions. This future research will reveal what needs to happen in places where $\mathrm{AC}$ behaviour is not yet occurring. For AC policies to be successful, designation is perhaps necessary, but it is not sufficient.

\section{References}

Cervero, R. (1995). Planned communities, self-containment and commuting: Across-national perspective. Urban Studies, 32(7), 1135-61. doi.org/10.1080/00420989550012618

Cervero, R. \& Landis, J. (1991). Suburbanization of jobs and the journey to work. UC Berkeley: University of California Transportation Center.

Day, J., Han, W., Wu, A.B. \& Zheng, J.R. (2017). Does policy invoke sub-centres? Econometric evaluation of Melbourne's urban spatial planning since 1996. (Working Paper). 
Day, J., Sturup, S., Chen, Y., Budahazy, M., Wu, A. \& Fan, L. (2015). Activity centre policy effects on employment clustering: A spatial study of job density in Melbourne, Australia. Paper presented at the State of Australian Cities National Conference, Gold Coast, Queensland, Australia.

Giuliano, G. (1991). Is Jobs-Housing Balance a Transportation Issue? UC Berkeley: University of California Transportation Center.

Giuliano, G. \& Small, K.A. (1991). Subcenters in the Los Angeles Region. Regional Science and Urban Economics, 21(2), 163-82. doi.org/10.1016/0166-0462(91)90032-I

Goodman, R., Buxton, M., Chhetri, P.R., Taylor, E.R. \& Wood, G.R. (2010). Planning and the characteristics of housing supply in Melbourne. Melbourne: Australian Housing and Urban Research Institute (AHURI).

Gordon, P., Richardson, H.W. \& Jun, M.J. (1991). The commuting paradox evidence from the top twenty. Journal of the American Planning Association, 57(4), 416-20. doi.org/10.1080/01944369108975516

Kiviet, J.F. (1995). On bias, inconsistency, and efficiency of various estimators in dynamic panel data models. Journal of Econometrics, 68, 53-78. doi.org/10.1016/0304-4076 (94)01643-E

Lin, D., Allan, A. \& Cui, J. (2015). The impact of polycentric urban development on commuting behaviour in urban China: Evidence from four sub-centres of Beijing. Habitat International, 50, 195-205. doi.org/10.1016/j.habitatint.2015.08.018

Mees, P. (2003). Paterson's Curse: the attempt to revive metropolitan planning in Melbourne. Urban Policy and Research, 21(3), 287-99. doi.org/10.1080/0811114032000113671

Melbourne and Metropolitan Board of Works (1981). Metropolitan Strategy Implementation. Melbourne.

Monaghan, A., Burke, M. \& Pan, H. (2015). The Shanghai Model for Activity Centres and its Potential in the Australian Context. Paper presented at the State of Australian Cities National Conference, Gold Coast, Queensland, Australia.

Peng, Z. R. (1997). The jobs-housing balance and urban commuting. Urban Studies, 34(8), 1215-35. doi.org/10.1080/0042098975600

Productivity Commission (2011). Economic structure and performance of the Australian retail industry. Canberra: Commonwealth of Australia.

Schleith, D., Widener, M. \& Kim, C. (2016). An examination of the jobs-housing balance of different categories of workers across 26 metropolitan regions. Journal of Transport Geography, 57, 145-60. doi.org/10.1016/j.jtrangeo.2016.10.008

Victorian Department of Infrastructure (2002a). Melbourne 2030: Planning for Sustainable Growth. Melbourne. 
Victorian Department of Infrastructure (2002b). Melbourne 2030: Planning for sustainable growth-Draft Implementation Plan 4 Activity Centres. Melbourne.

Victorian Department of Planning and Community Development (2010). Structure Planning for Activity Centres, Practice Notes. Melbourne.

Victorian Department of Planning and Development (1995). Living Suburbs: A Policy for Metropolitan Melbourne into the 21st Century. Melbourne.

Victorian Department of Transport (2014). Plan Melbourne: Metropolitan Planning Strategy. Melbourne. 
This text is taken from Agenda, Volume 25 - Number 1, 2018, edited by William Coleman, published 2018 by ANU Press, The Australian National University, Canberra, Australia.

doi.org/10.22459/AG.25.01.2018.01 